\title{
Persuasión en el interrogatorio policial: una revisión sistemática
}

Persuasion in police interview: a Systematic Review

\author{
María Navamuel Nieto ${ }^{1}$ \\ Rafael Manuel López Pérez ${ }^{2}$ \\ (1) Investigadora independiente, Madrid, España. \\ (2) Fundación Universitaria Behavior \& Law, Madrid, España. \\ Email correspondencia:mnavamuel@gmail.com
}

\section{Resumen}

El interrogatorio y la entrevista policial son unos de los elementos más importantes en la investigación criminal. Uno de sus principales objetivos es obtener información veraz tanto de sospechosos como de testigos que sirva para esclarecer el delito investigado. Para conseguir ese objetivo, diversas técnicas de persuasión son utilizadas en función del tipo de interrogatorio que se aplique. En esta revisión teórica se ha adoptado un proceso de revisión sistemática mediante el cual se recopilarán y analizarán estudios procedentes de dos bases de datos diferentes, planteando los factores que influyen en el proceso de interrogatorio y relacionándolos con las técnicas de persuasión identificadas en estos y sus potenciales riesgos.

Palabras Clave: Persuasión, interrogatorio policial, entrevista policial, influencia, confesiones, investigación policial

\begin{abstract}
Police interrogation and police interview are two of the most relevant factors in criminal investigation. One of their main goals is to obtain complete and accurate information both from suspects and witnesses in order to clarify the crime investigated. Several persuasion techniques are used depending on the type of interrogation applied. This theoretical revision has adopted a systematic review method where articles will be chosen, and then analyzed, from two different databases. Factors influencing the interrogation process will be presented and connected with the identified persuasion techniques and their potential risks.
\end{abstract}

Keywords: Persuasion, police interrogation, police interview, influence, confessions, police investigation

Fecha de envío: 18/03/2017

Fecha de aceptación: 13/04/2017 


\section{INTRODUCCIÓN}

Persuadir según el diccionario de la lengua española es el acto de " inducir, mover, obligar a alguien con razones a creer o hacer algo" ("Diccionario de la lengua española (RAE)," 2016). Se han estudiado las técnicas de persuasión e influencia en diferentes ámbitos y con diferentes objetivos en el acto de persuadir, como por ejemplo, conseguir el voto en política, obtener la venta en marketing, etc. Cialdini (1984), en un libro de referencia, profundiza en principios de la influencia utilizados en diferentes ámbitos como son la reciprocidad, la escasez, la autoridad, el compromiso y coherencia, la prueba social o consenso y la simpatía.

En el ámbito policial el principal objetivo que busca la persuasión en un interrogatorio con un sospechoso de un delito, es la obtención de información veraz relacionada con un hecho delictivo (Choe, 2013; Walton, 2003). Sin embargo, se pueden encontrar objetivos contrapuestos en el caso del agente de policía y el sospechoso del delito. Mientras el interrogador buscará obtener la mayor cantidad de información y evidencias posibles, el interés del interrogado puede ser el contrario (Gaines, 2011; Walton, 2003).

La manera en que muchos de los interrogatorios están diseñados (búsqueda de pruebas para incriminar a los culpables) pueden tener efectos diferentes a los esperados. Los métodos de interrogatorio pueden llevar a obtener falsas confesiones y por añadidura, condenas erróneas (Kassin, Drizin, Grisso, Gudjonsson, Leo, \& Redlich, 2010; Madon, Guyll, Scherr, Greathouse, \& Wells, 2012). Las confesiones han probado tener mayor impacto en las decisiones de un jurado que otros elementos como pruebas físicas o el testimonio de testigos (Beauregard, Deslauriers-Varin, \& St-Yves, 2010; Kassin \& Neumann, 1997). Se entiende por interrogatorio aquel tipo de entrevista mediante la cual el interrogador imputa al sospechoso su implicación en un hecho delictivo. Al mismo tiempo, también será interesante el uso de la persuasión en otro tipo de entrevista: la entrevista de investigación. En este caso, se habla del proceso en el que dos o más personas mantienen una conversación destinada a recopilar información para esclarecer un hecho concreto (González, 2009). En ésta será crítica la obtención de información y evidencias para el delito investigado. El tratamiento en el ámbito policial es diferente entre ambos casos (Vrij, Mann, \& Fisher, 2006). Existen además diferencias en los estilos de interrogatorio en función del país que se trate. Se encuentra por ejemplo un ejemplo de interrogatorio más "acusatorio" en Estados Unidos (basado en la técnica Reid de entrevista e interrogatorio (Inbau, Reid, Buckey, \& Jayne, 2001)), mientras que en lugares como Inglaterra y Gales el estilo predominante es el de "obtención de información" (Vrij et al., 2006).

Este último caso está influido por la normativa establecida en Gran Bretaña desde 1984 con la Police and Criminal Evidence Act (PACE) que pretende regular, entre otros aspectos, la relación con los sospechosos en el proceso de interrogatorio para así proteger los derechos de estos y tener las mayores garantías posibles en los testimonios y confesiones obtenidas (Police and Criminal Evidence Act (PACE), 1984).

\section{A. Justificación de la revisión.}

En esta revisión sistemática se tratará de obtener una visión global del proceso de entrevista-interrogatorio policial analizando los efectos de la persuasión y de las diferentes técnicas empleadas para la obtención de la confesión de los culpables o de información relevante por parte de los testigos. Se revisarán los efectos que determinados estilos de interrogatorio provocan sobre los entrevistados (como por ejemplo, falsas confesiones) en función de diferentes factores y se plantearán posibles vías de avance en la investigación. Todo ello contribuirá a tener una visión amplia de las investigaciones científicas en el campo, ordenando el conocimiento y pudiendo así determinar futuros focos de investigación para avanzar en iniciativas relevantes que necesitan de una mayor exploración.

\section{MÉTODO}

Se describirán aquí los métodos utilizados para la realización de la revisión sistemática y garantizar así su replicabilidad.

\section{A. Criterios de búsqueda}

Para la realización de la revisión teórica se seleccionaron dos bases de datos científicas: Science Directy PubMed.

Science Direct. ofrece artículos en texto completo escritos por investigadores de renombre internacional. Da acceso a 1.800 revistas científicas Elsevier, con más de 9 millones de artículos en las áreas científica, tecnológica y médica, 
representando aproximadamente $25 \%$ de la producción científica mundial (Science Direct, 2016).

PubMed: es un motor de búsqueda de libre acceso a la base de datos MEDLINE de citaciones y resúmenes de artículos de investigación biomédica. Ofrecido por la Biblioteca Nacional de Medicina de los Estados Unidos como parte de Entrez. MEDLINE tiene alrededor de 4800 revistas publicadas en Estados Unidos y en más de 70 países de todo el mundo desde 1966 hasta la actualidad (PubMed, 2016).

Como criterios generales de la búsqueda y cribado de información se han utilizado los siguientes:

- Se han seleccionado sólo artículos científicos (papers).

- Se ha buscado siempre en primer lugar en la base de datos de Science direct y con posterioridad en la base de datos de PubMed eliminando de la selección aquellos artículos ya disponibles en búsquedas anteriores.

- Los tesauros utilizados para la búsqueda han sido los siguientes para cubrir el mayor número de artículos posible relativos a persuasión (o influencia) en interrogatorio policial (o entrevista policial):

o Influence and "police interview"

o Persuasion and "police interview"

- Influence and "police interrogation"

- Persuasion and "police interrogation"

- Se ha decidido seleccionar artículos científicos en inglés, francés o español resultantes de la búsqueda.

- Se han acotado las búsquedas en la base de datos de Science Direct por temas relevantes (detallados en Tabla 1)

- Se han descartado en la selección final aquellos artículos que en el título o en el abstract no hubiese mención o relación con la entrevista o interrogatorio policial así como aquellos referidos a investigaciones aplicadas a menores por sus diferentes características. La búsqueda se realizó en Julio de 2016.

A continuación se muestran las búsquedas, resultados obtenidos y número de artículos seleccionados finalmente para cada base de datos científica.

\section{B. Detalles de búsquedas}

Tabla 1. Detalle de las búsquedas

\begin{tabular}{|c|c|c|c|c|}
\hline $\begin{array}{l}\text { POLICE } \\
\text { INTERVIEW }\end{array}$ & Búsqueda & $\begin{array}{l}\text { Science Direct } \\
\text { (artículos) }\end{array}$ & $\begin{array}{l}\text { PubMed } \\
\text { (artículos) }\end{array}$ & Totales \\
\hline \multirow{3}{*}{$\begin{array}{l}\text { Búsqueda } 1 \\
\text { (B1) }\end{array}$} & influence and "police interview" & 183 & 7 & 190 \\
\hline & $\begin{array}{l}\text { Refinada con } \\
\text { "police, interview, forensic" }\end{array}$ & 38 & n.a. & 38 \\
\hline & Selección B1 & 20 & 2 & 22 \\
\hline \multirow[t]{3}{*}{$\begin{array}{l}\text { Búsqueda } 2 \\
\text { (B2) }\end{array}$} & $\begin{array}{l}\text { Persuasion and "police } \\
\text { interview" }\end{array}$ & 17 & 6 & 23 \\
\hline & $\begin{array}{l}\text { Refinada con "police,false } \\
\text { confession,suspect,cognitive } \\
\text { interview,criminal behavior" }\end{array}$ & 9 & n.a. & 9 \\
\hline & Selección B2 & 1 & 4 & 5 \\
\hline $\begin{array}{l}\text { Total police } \\
\text { interview } \\
(\mathbf{B 1 + B 2}) \\
\end{array}$ & Selección B1+B2 & 21 artículos & 6 artículos & 27 artículos \\
\hline
\end{tabular}

Resultados de las búsquedas Influence and "police interview" y Persuasion and "police interview" y selección artículos. 


\begin{tabular}{|c|c|c|c|c|}
\hline $\begin{array}{l}\text { POLICE } \\
\text { INTERROGATION }\end{array}$ & Búsqueda & $\begin{array}{l}\text { Science } \\
\text { Direct } \\
\text { (artículos) }\end{array}$ & $\begin{array}{l}\text { PubMed } \\
\text { (artículos) }\end{array}$ & Totales \\
\hline \multirow[t]{3}{*}{ Búsqueda 3 (B3) } & Influence and police interrogation & 117 & 0 & 117 \\
\hline & $\begin{array}{l}\text { Refinada con "police, false } \\
\text { confession, interview,suspect,criminal, individual } \\
\text { difference" }\end{array}$ & 28 & n.a. & 28 \\
\hline & Selección B3 & 5 & 0 & 5 \\
\hline \multirow[t]{2}{*}{ Búsqueda 4 (B4) } & Persuasion and police interrogation & 17 & 1 & 18 \\
\hline & Selección B4 & 2 & 1 & 3 \\
\hline $\begin{array}{l}\text { Total Police } \\
\text { interrogation } \\
(\mathrm{B} 3+\mathrm{B} 4) \\
\end{array}$ & Selección B3+B4 & $\begin{array}{l}7 \\
\text { artículos }\end{array}$ & $\begin{array}{l}1 \\
\text { artículo }\end{array}$ & $\begin{array}{l}8 \\
\text { artículos }\end{array}$ \\
\hline $\begin{array}{l}\text { Total } \\
(\mathrm{B} 1+\mathrm{B} 2+\mathrm{B} 3+\mathrm{B} 4)\end{array}$ & Selección B1+B2+B3+B4 & $\begin{array}{l}28 \\
\text { artículos }\end{array}$ & $\begin{array}{l}7 \\
\text { artículos }\end{array}$ & $\begin{array}{l}35 \\
\text { artículos }\end{array}$ \\
\hline
\end{tabular}

Resultados de las búsquedas Influence and "police interrogation" y Persuasion and "police interrogation" y artículos totales seleccionados.

Como se puede ver en esta tabla, se han seleccionado un total de 35 artículos para la revisión de los cuales 28 corresponden a la base de datos de Science Direct y 7 han sido seleccionados en la base de datos de PubMed.

En la figura 1 se muestra el reparto entre las bases de datos. En la tabla 3 en el anexo se muestra la relación de artículos seleccionados con sus autores.

Una vez ya realizada la criba y selección final de artículos aceptados, se ha procedido a la ordenación de la información obtenida, que se tratará a continuación.

\section{Figura 1. Artículos seleccionados (\%)}

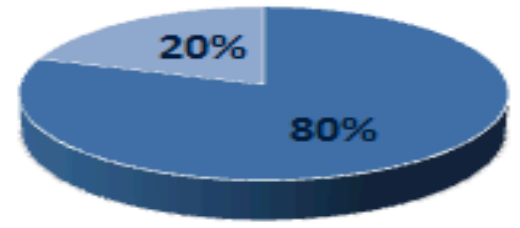

- Science Direct $=$ PubMed

Se muestran en la Figura 1 el porcentaje de artículos de la revisión correspondientes a cada base de datos científica. El $80 \%$ de los artículos pertenece a Science Direct

\section{RESUltados}

Se abordarán los resultados pasando por las diferentes fases que pueden formar parte de un proceso de entrevista policial, que son: pre-entrevista $o$ valoración previa sobre la necesidad y adecuación de la persona para ser entrevistada, entrevista y confesión ( $\mathrm{y}$ sus efectos en el procesamiento y condena). En la entrevista se analizarán los métodos de persuasión y el impacto que pueden tener en la información obtenida y en la propia confesión del entrevistado.

\section{A. Pre-entrevista y adecuación del sujeto para la entrevista}

Antes de pasar a la fase de interrogatorio o entrevista policial existen dos prácticas a tener en cuenta:

- La pre-entrevista para determinar sin el sujeto merece ser o no sometido al interrogatorio.

- La valoración de las condiciones físicas y mentales del sujeto para poder ser interrogado con garantías.

1) Pre-entrevista

Según la técnica Reid de interrogatorio (Inbau et al., 2001) que se analizará más adelante- muy difundida en Norteamérica- en muchas ocasiones, antes de iniciar un proceso de interrogatorio se produce con el sujeto un proceso de pre-entrevista en el que se trata de obtener información previa que permita valorar la necesidad o no de interrogar. El principal objetivo de este proceso es valorar si el sujeto es inocente o culpable. Este proceso puede sostenerse con información obtenida de testigos 
u otras pruebas, pero también de valoraciones sobre comportamiento verbal o actitudes de los sospechosos o de su respuesta a preguntas "generadoras de comportamientos" cuya respuesta puede predecir la culpabilidad o inocencia de la persona (p.e. "¿qué debería pasarle a quien cometió el crimen?"). Los interrogadores con el entrenamiento podrían llegar a detectar la mentira con una precisión del 85\% (Inbau et al., 2001). Sin embargo este nivel de detección de la mentira excede con mucho la media detectada en investigaciones en el mundo (Kassin \& Gudjonsson, 2004). Es por este motivo que la pre-entrevista en esos términos como elemento decisor de llevar a cabo un interrogatorio es un elemento que generará errores debido a su parcialidad y subjetividad (Kassin \& Gudjonsson, 2004).

\section{2) Adecuación para la entrevista}

La valoración de la adecuación para la entrevista trata de evitar los riesgos de obtener información no fiable del sospechoso y de garantizar la competencia del mismo para llevar a cabo el proceso de interrogatorio. Condiciones consideradas de riesgo son la embriaguez temporal, intoxicación por drogas, síndrome de abstinencia, cansancio o dolor físico severos, dolencia física grave o enfermedad mental severa susceptibles de ser tratadas, y las condiciones permanentes de demencia o discapacidad mental graves (Gall \& Freckelton, 1999). En un estudio realizado por Gall \& Freckleton (1999) el $35 \%$ de los sujetos que fue evaluado por los riesgos de estar bajo los efectos del alcohol o drogas o por cuestiones psiquiátricas, fueron considerados no aptos para ser interrogados y de los considerados aptos, un $14 \%$ lo fue siempre que hubiese una tercera parte presente en el interrogatorio. Decidir si es necesaria una tercera persona independiente durante la entrevista es otra de las tareas a realizar en la evaluación de la adecuación de la persona para la entrevista (Gall \& Freckelton, 1999).

Aunque los efectos de las drogas o el alcohol en los sujetos durante el interrogatorio en la custodia policial no son plenamente conocidos, sí hay evidencias que manifiestan que los sujetos bajo estos efectos pueden ver reducida su capacidad para pensar y recordar claramente y por tanto, dar información equivocada a la policía. Sin embargo, estar bajo los efectos de las drogas o alcohol no parece incrementar el nivel de ansiedad del interrogado o influenciar su necesidad de confesar
(Davison \& Gossop, 1999; Sigurdsson \& Gudjonsson, 1994).

No todos los detenidos colaboran con la evaluación previa que realiza el médico forense (posiblemente debido a la asociación que los detenidos pueden realizar de este con la policía) lo que no impide que el médico deba emitir su examen. Por ello es fundamental el entrenamiento de estos profesionales para poder realizar dictámenes objetivos y ajustados en cada caso (Gall \& Freckelton, 1999).

\section{B. La entrevista e interrogatorio policial}

La entrevista policial es uno de los elementos críticos de la investigación policial ayudando a construir el caso con las pruebas obtenidas de víctimas, testigos y del propio sospechoso. Dado que la confesión es un punto muy relevante para la construcción del caso, los investigadores recurren a diversos métodos para obtenerla (coercitivos, engaño, etc.) (Choe, 2013)

Aunque el foco principal del interrogatorio ha sido la obtención de la confesión, desde los años 90 en adelante y especialmente en Inglaterra, este se ha trasladado hacia la obtención de información fiable más que a la confesión en sí (Gudjonsson, 2006).

Así mismo, además de la entrevista al sospechoso, existe la entrevista a los testigos cooperativos en la que la obtención de la máxima información posible es crítica. Sin embargo, los agentes policiales reciben menos entrenamiento para desarrollar este tipo de entrevistas, lo que resulta en la obtención de una menor cantidad de información además de no ayudar a las víctimas a mitigar el impacto psicológico que el crimen ha podido generarles (Ronald P. Fisher \& Geiselman, 2010).

Se mostrarán a continuación dos tipologías de entrevista representativas de ambas orientaciones: interrogatorio orientado a la confesión, entrevista cognitiva orientada a la obtención de la máxima información.

1) Interrogatorio orientado a la confesión

En este tipo de interrogatorio las tácticas incluyen desde apelar al propio interés del acusado como a confrontaciones, acusaciones o cierta negociación (Mason, 2016). Buscan también hacer olvidar al acusado las consecuencias que a largo plazo puede tener su confesión (Madon et al., 2012). 
Un ejemplo de este tipo de interrogatorio extendido fundamentalmente en Norteamérica y de gran influencia es la técnica Reid (Inbau et al., 2001). El modelo Reid asume que todos aquellos que han sido seleccionados para ser interrogados son culpables y sólo confesarán en el caso de que las consecuencias percibidas de esa confesión sean más atrayentes que la ansiedad generada por mantener su mentira. El procedimiento persigue la autoinculpación del sospechoso aumentando la ansiedad y la desesperación y minimizando las consecuencias de la confesión (Kassin \& Gudjonsson, 2004).

Los nueve pasos que plantea la técnica Reid (Inbau et al., 2001) son:

Antes de nada, el interrogatorio debe llevarse a cabo en una estancia pequeña, sin apenas mobiliario y aislada acústicamente dentro de la comisaría de policía. Así se consigue el aislamiento del sospechoso de entornos familiares y personas conocidas aumentando su ansiedad e incomodidad, que le llevarán a tratar de escapar de esa situación. Para aumentar su incomodidad se sentará al sospechoso en sillas duras, sin reposabrazos, lejos de los controles de temperatura ambiental y tratando, a lo largo del interrogatorio, de invadir su espacio personal. Recomienda, además, que en la sala exista un espejo de una dirección para poder observar cambios en el interrogado. Una vez establecido lo anterior se ponen en marcha los 9 pasos:

Paso 1: el interrogador se enfrenta al sospechoso con firmes aseveraciones sobre su culpabilidad.

Paso 2: después desarrolla argumentos que justifican o excusan psicológicamente el crimen.

Paso 3: interrumpe cualquier esfuerzo del acusado en negarlo o en defenderse.

Paso 4: debilita y derrota las objeciones emocionales, morales o de hecho de los sospechosos.

Paso 5: Se asegura de que un sospechoso pasivo no se retracte.

Paso 6: muestra empatía y comprensión y apremia al sospechoso para que coopere.

Paso 7: sobre el acto que se investiga le ofrece al sospechoso una alternativa que le permite salvar las apariencias.

Paso 8: hace que el sospechoso vuelva a contar los detalles de su crimen.
Paso 9: convierte la declaración anterior en una confesión completa oral o escrita.

Estos pasos pueden concretarse en tres (Kassin \& Gudjonsson, 2004): aislamiento, que incrementa la ansiedad del sospechoso por evitarlo; confrontación mediante la que el interrogador acusa al sospechoso presentándole pruebas y negando cualquier objeción de este; minimización en la que el interrogador se muestra comprensivo y minimiza las consecuencias de la confesión del crimen.

Los creadores de la técnica Reid (Inbau et al., 2001) defienden el método para obtener confesiones de los culpables y niegan que pueda generar confesiones falsas en sujetos inocentes. Sin embargo, no aportan pruebas o evidencia científica de esto último. Por el contrario, muchos científicos manifiestan su preocupación sobre las técnicas de muchos manuales actuales de interrogatorio (no sólo el de la técnica Reid) que pueden llevar a obtener falsas confesiones (Gudjonsson, Sigurdsson, Sigfusdottir, \& Asgeirsdottir, 2008; Kassin \& Neumann, 1997; Russano, Meissner, Narchet, \& Kassin, 2005).

2) Entrevista para la obtención de información (cognitiva o investigativa)

Aunque en países como Reino Unido, Suecia o Australia el entrenamiento de los agentes policiales en la entrevista a testigos cooperativos está más implantado, sigue siendo una cuestión secundaria en el resto del mundo (Fisher \& Geiselman, 2010).

Sin embargo, sí existe una amplia literatura sobre la entrevista cognitiva y sus técnicas. Esta consiste en un protocolo de entrevista no directivo basado en recuperación de recuerdos procedente de teorías de la memoria. En términos generales se trata de un recuerdo libre -respetando los procesos mentales del testigo-, sin interrupciones del investigador, que permite al testigo describir elementos específicos de un momento del crimen. Para ello, será importante el entrenamiento del investigador, la flexibilidad y adaptación de las preguntas a las características mentales del testigo para favorecer el recuerdo y el preguntar por el evento más de una vez a lo largo de la entrevista (Launay \& Py, 2015).

La entrevista cognitiva fue desarrollada por Ron Fisher and Ed Geiselman (Fisher, Geiselman, \& Amador, 1989). Esta tiene cuatro componentes (Yuille, Marxsen, \& Cooper, 1999): 
1. Reinstalación del contexto: el testigo debe recrear mentalmente el contexto en el que el evento ocurrió (tiempo, actividad previa, etc.).

2. Recuerdo exhaustivo: se le pide al testigo relatar cualquier recuerdo asociado, sin importar si parece o no relevante.

3. Cambio de perspectiva: se le pide al testigo que recuerde el evento desde una perspectiva diferente a la que él tenía (p.e. como si lo viera en video).

4. Recuerdo hacia atrás: al testigo se le pide recordar el evento en orden temporal inverso.

En resumen, el interrogador para llevarla a cabo correctamente debe tener en cuenta el impacto en la declaración del testigo que pueden ejercer aspectos como la diversidad cultural, la de su propia personalidad, sus motivos o el rol e impacto del evento (Yuille et al., 1999).

Las habilidades que un interrogador tendrá que desarrollar para realizar entrevistas más efectivas serán (Yuille et al., 1999):

Mente crítica y abierta para llevar a cabo un proceso de investigación efectivo.

Rapport, para generar confianza y comodidad en el testigo en el proceso.

Habilidades de escucha para no interrumpir al testigo en su relato y ayudar a que este fluya.

Técnicas de formulación de preguntas, para hacer preguntas abiertas y no directivas al principio y dejar las cerradas necesarias para más adelante.

Personalidad, favoreciendo aspectos de esta que faciliten la relación de confianza con el testigo para la obtención de información.

El entrenamiento y la práctica continuada en este tipo de entrevista son fundamentales y permitirán extraer todas las ventajas que esta técnica proporciona: más información de testigos, posible salvaguarda ante falsos testimonios por la no presunción de culpabilidad, etc. (Vrij et al., 2006; Yuille et al., 1999).

Se ha detectado también que, en determinadas circunstancias la entrevista colectiva de estas características puede hacer aflorar más recuerdos que las realizadas individualmente con las mismas personas (Vernham, Vrij, Leal, Mann, \& Hillman, 2014).
3) El silencio en la entrevista y contrainterrogatorio

El silencio o el derecho a permanecer en silencio, en general, es difícil que se considere como una respuesta apropiada a una pregunta o a una acusación. A pesar de que en un entorno policial el derecho a permanecer en silencio debe preservarse, existe el riesgo de que los interrogadores hagan asunciones sobre la culpabilidad de quien no niega una acusación. En última instancia, este derecho está garantizado en los tribunales, ya que no se permiten inferencias adversas sobre un acusado por el hecho de que ejerza su derecho a permanecer en silencio (Heydon, 2011).

En países como China, sin embargo, la evolución del sistema judicial junto con la tradición cultural e idiosincrasia del país, que considera la confesión como el primer paso a la redención de la culpa, no incluye en su sistema legal (y existen barreras a que se incluya) el derecho a permanecer en silencio (Wu \& Vander Beken, 2012).

Hay que tener en cuenta también que el silencio en el interrogatorio puede tener otras causas tales como proteger a un familiar, no querer desvelar cierta información potencialmente embarazosa para el testigo, etc. (Rix, 1998). También se pueden dar situaciones en las que la entrevista se realiza a sospechosos de otro país que necesitan intérprete, con lo que determinados silencios en la conversación y en la traducción serán difíciles de interpretar correctamente (influyendo incluso en la entrevista las propias convicciones del intérprete) (Nakane, 2011).

El silencio además puede ser utilizado como una táctica en un contrainterrogatorio. Además de esta otras tácticas de contrainterrogatorio han sido estudiadas (Alison, Alison, Noone, Elntib, Waring, \& Christiansen, 2014):

Pasiva: permanecen en silencio, no miran a entrevistador. Usada principalmente por paramilitares y terroristas internacionales.

Pasivo verbal: respuesta monosilábica, hablan de falta de memoria.

Verbal: hablan de un tema no relacionado, dan información ampliamente conocida y además se retractan de declaraciones anteriores además de usar el recurso de "sin comentarios". Usado por paramilitares principalmente. 
4) Factores de persuasión en la entrevista

Como se ha visto en las técnicas anteriores existen diversos factores que influyen de una u otra manera en la obtención de información o en la confesión del sospechoso. Se identifican aquí elementos de persuasión que van a influir en el proceso de entrevista:

Maximización y minimización (Kassin et al., 2010; Russano et al., 2005). En la maximización se encuentran tácticas coercitivas que persiguen intimidar al sospechoso (confrontación, negación de las objeciones, presentando pruebas fabricadas, largos interrogatorios, aislamiento, etc.). La minimización busca disminuir la importancia del delito a ojos del sospechoso, tratando de empatizar con él y ofreciéndole salidas que le permitan salvar las apariencias. Además, muchos sospechosos asumen que la minimización implica tácitamente una promesa de indulgencia. El uso de la minimización incrementa también, según estudio de Russano et al. (2005), las confesiones tanto en culpables como en inocentes, por lo que, al igual que las tácticas coercitivas, estas deben ser evitadas (especialmente la "promesa" de indulgencia en la práctica del interrogatorio). En los casos más graves determinadas técnicas de maximización como el aislamiento, largos interrogatorios, etc. pueden provocar el "síndrome de desconfianza en la memoria" en los que el testigo llega a creer la versión que el interrogador le presenta incluso cuando realmente los hechos que él mismo ha experimentado no se han producido así (Gudjonsson, 2016).

Estos mismos factores que tratan de llevar al acusado a la confesión se pueden agrupar en (Gudjonsson, Sigurdsson, Asgeirsdottir, \& Sigfusdottir, 2006): intimidación (incrementando ansiedad del sospechoso respecto a su negación), desafío robusto (desafiando mentiras e inconsistencias) y manipulación (justificando o excusando el delito). Las dos primeras formarían parte de las técnicas de maximización y la última correspondería a la minimización.

Uso de técnicas sensitivas como el "apelar a la consciencia del sospechoso" o el "desafío suave" (Gudjonsson et al., 2006): de mayor efectividad en delincuentes sexuales, consisten en apelar al buen carácter del acusado para que diga la verdad o en el caso del desafío suave, presentan la versión de los hechos del sujeto empleando un tono bajo y mostrando evidencias de manera que reduzcan la sensación de vergüenza. No se ha probado que estas técnicas menoscaben la admisibilidad de la confesión.

Acentuando las consecuencias a corto plazo vs las de largo plazo (Madon et al., 2012). Los estudios demuestran que las personas se rigen en sus decisiones preferentemente por las consecuencias de sus actos a corto plazo frente a lo que pueda suceder más adelante. Este conocimiento es usado en las técnicas de interrogatorio que persiguen la confesión rápida del interrogado mediante tácticas como la incomodidad en el proceso, la larga duración de las entrevistas y la minimización de las consecuencias futuras de la confesión. Estas tácticas tienen también efecto en personas inocentes que pueden llevar a falsas confesiones, otro de los motivos por los que esta técnica no es recomendable en los procesos de entrevista.

Usando el feedback negativo y preguntas directivas para alterar las declaraciones $y$ percepciones de los interrogados. El feedback negativo es una influencia que puede resultar en los entrevistados en una sensación de que sus respuestas son erróneas o hay algo malo en ellos mismos. Este sentimiento puede llevar (en función de la tipología del entrevistado) a cambiar declaraciones previas. Ejemplos de feedback negativo pueden ser mensajes de que el entrevistado ha cometido algún error o miente, menciones negativas sobre su persona o incluso la mera repetición de preguntas ya realizadas y contestadas por el entrevistado (McMurtrie, Baxter, Obonsawin, \& Hunter, 2012). El uso del feedback negativo puede generar incertidumbre, disminuir la autoestima o incrementar la ansiedad en los sujetos, lo que les hace más vulnerables a la sugestión y con menor confianza en su propia experiencia, lo que finalmente puede resultar en cambios en su testimonio (Baxter, Boon, \& Marley, 2006).

El feedback negativo tiene efectos significativos en el estado de ánimo y en la capacidad de sugestión del sujeto. Generalmente estos efectos se manifiestan en dos vertientes: en primer lugar hacen a los sujetos cambiar sus respuestas previas y en segundo lugar incrementan su susceptibilidad ante futuras preguntas directivas, pudiendo mantenerse estos efectos en el tiempo. El feedback positivo, por el contrario, refuerza una respuesta previa (Tata \& Gudjonsson, 1990).

El uso de preguntas directivas (p.e. preguntas que 
llevan implícita una respuesta que es la correcta) introducen también detalles inexactos en la investigación, por lo que se debe ser vigilante para evitar que aparezcan (Baxter et al., 2006).

Dados los riesgos asociados al feedback negativo y a las preguntas directivas que pueden desembocar en confesiones falsas o en obtención de información inexacta en una investigación, deberán tomarse las precauciones para que no estén presentes en el interrogatorio (Baxter et al., 2006; McMurtrie et al., 2012; Tata \& Gudjonsson, 1990).

Existen otros factores o habilidades persuasivas más orientados a la obtención de información mencionados ya en el apartado de entrevista cognitiva (Yuille et al., 1999):
Mente crítica y abierta para llevar a cabo un proceso de investigación efectivo.

Establecer rapport, para generar confianza y comodidad en el testigo en el proceso.

Escucha activa que no interrumpa al testigo en su relato $y$ ayude a que este fluya sin intervenciones directivas.

Formulación de preguntas abiertas y no directivas, sobre todo al principio, dejando las preguntas cerradas para concretar algún detalle al final.

Personalidad, favoreciendo aspectos de esta que faciliten la relación de confianza con el testigo para la obtención de información.

Tabla 2. Factores persuasión, tipo de entrevista y fiabilidad de la información

\begin{tabular}{|c|c|c|c|c|c|c|c|c|c|}
\hline $\begin{array}{l}\text { Factores } \\
\text { Persuasión }\end{array}$ & $\begin{array}{l}\text { Mente } \\
\text { crítica } \\
\text { abierta }\end{array}$ & Rapport & Escucha & $\begin{array}{l}\text { Preguntas } \\
\text { abiertas }\end{array}$ & Personalidad & $\begin{array}{l}\text { Maximización y } \\
\text { minimización }\end{array}$ & $\begin{array}{l}\text { Feedback } \\
\text { negativo, } \\
\text { preguntas } \\
\text { directivas }\end{array}$ & $\begin{array}{l}\text { Técnicas } \\
\text { sensitivas }\end{array}$ & $\begin{array}{l}\text { Corto } \\
\text { plazo vs } \\
\text { Largo } \\
\text { plazo }\end{array}$ \\
\hline $\begin{array}{c}\text { Tipo } \\
\text { entrevista } \\
\text { preferente } \\
\text { (orientada } \\
\text { información, } \\
\text { orientada } \\
\text { confesión) }\end{array}$ & $\begin{array}{l}\text { Orientada } \\
\text { información }\end{array}$ & $\begin{array}{l}\text { Orientada } \\
\text { información }\end{array}$ & $\begin{array}{l}\text { Orientada } \\
\text { información }\end{array}$ & $\begin{array}{l}\text { Orientada } \\
\text { información }\end{array}$ & $\begin{array}{l}\text { Orientada } \\
\text { información }\end{array}$ & $\begin{array}{l}\text { Orientada } \\
\text { confesión }\end{array}$ & $\begin{array}{l}\text { Orientada } \\
\text { confesión }\end{array}$ & $\begin{array}{l}\text { Orientada } \\
\text { confesión }\end{array}$ & $\begin{array}{l}\text { Orientada } \\
\text { confesión }\end{array}$ \\
\hline $\begin{array}{c}\text { Riesgo de } \\
\text { no fiabilidad } \\
\text { información }\end{array}$ & Menor riesgo & Menor riesgo & $\begin{array}{l}\text { Menor } \\
\text { riesgo }\end{array}$ & Menor riesgo & Menor riesgo & Mayor riesgo & Mayor riesgo & $\begin{array}{l}\text { Menor } \\
\text { riesgo }\end{array}$ & $\begin{array}{l}\text { Mayor } \\
\text { riesgo }\end{array}$ \\
\hline
\end{tabular}

En la tabla 2 se muestra una tabla resumen de factores persuasivos y la fiabilidad de la información que pueden obtener de los interrogados.

\section{La confesión en el interrogatorio}

En un estudio llevado a cabo entre los estudiantes de secundaria de la población de Islandia (Gudjonsson et al., 2006) un 53\% de aquellos que habían sido interrogados por la policía reconocieron haber realizado una confesión verdadera. Del resto de interrogados, un 7,6\% reconoció haber realizado una confesión falsa.

En otro estudio (Sigurdsson \& Gudjonsson, 1994) que investigaba el efecto de la intoxicación por drogas o alcohol y del síndrome de abstinencia en sospechosos criminales y sus confesiones, el ratio de confesión fue de un $92 \%$ (95\% entre los infractores graves de tráfico, $83 \%$ en los delincuentes sexuales).

Según Gudjonsson (2006), en Inglaterra la mayoría de delincuentes confesaban o hacían alguna admisión autoinculpadora.

Las principales razones para la confesión mostradas en los estudios se deben a una combinación de factores (Beauregard et al., 2010; Gudjonsson, 2006; Gudjonsson \& Petursson, 1991; Gudjonsson \& Sigurdsson, 1999; Sigurdsson \& Gudjonsson, 1994):

Percepción de la prueba. Es la percepción de la firmeza de las pruebas o evidencias en contra del sujeto que este tiene. Cuanto más consistentes son percibidas las pruebas contra el acusado, más predisposición existirá por parte de este para confesar.

Necesidad interna de confesar. Es la necesidad de quitarse "el peso de encima" contándolo todo. 
Presión externa. La presión que procede del exterior p.e. en la detención o la propia presión del interrogatorio.

En el estudio de Sigurdsson \& Gudjonsson (1994) se observó, además, que el hecho de que existiese un codemandando por el mismo delito, favorecía la decisión de confesar, poniendo al acusado en la tesitura del "dilema del prisionero".

En otra investigación (Beauregard et al., 2010), se observa que los delincuentes con antecedentes penales son más propensos a no confesar y consultar con un abogado (que les podrá recomendar permanecer en silencio) a la vez que conocen mejor las implicaciones a corto y a largo plazo que una confesión puede tener.

1) Confesión según la tipología del delito

En la mayoría de los casos la tipología del delito es una de las variables que menos pueden influir en la decisión de confesar si se compara, por ejemplo, con la de la percepción de las pruebas en contra del acusado. No obstante se han obtenido estos resultados en diversos estudios (Beauregard et al., 2010; Gudjonsson, 2006; Sigurdsson \& Gudjonsson, 1994):

- Delincuentes violentos, seguidos de violadores y abusadores de niños son los más impactados en su decisión de confesar por la percepción de la prueba contra ellos. Los acusados de abusos a menores fueron los más impactados por la necesidad interna de confesar, pero se resisten a ello posiblemente por los efectos de la vergüenza de confesar delitos sexuales. Por ello es importante en el interrogatorio tenerlo presente tratando de apelar a su consciencia y superar ese sentimiento para que confiesen (Gudjonsson, 2006).

- Según el estudio de Sigurdsson \& Gudjonsson, (1994) el 95\% de los delincuentes de tráfico del estudio y el $93 \%$ de los delincuentes de drogas confesaron a la policía (muchos de ellos fueron detenidos en el momento de la comisión del delito), mientras que en el caso de delincuentes sexuales la confesión se produjo en el $83 \%$ de los casos (en estos últimos la detención no suele producirse en el momento de la comisión del delito).

\section{2) Confesión según la personalidad}

Según el estudio de Gudjonsson \& Sigurdsson (1999) existe una correlación significativa entre las características de la personalidad del sujeto y las razones para confesar. La predisposición a la ansiedad (neuroticismo) y conformidad o conformismo como rasgos de la personalidad, están significativamente correlacionados con la necesidad interna de confesar y en mucha menor medida con la presión externa. La necesidad interna de confesar está relacionada también con dificultades en la confesión relacionados con la vergüenza que produce el delito.

En el estudio de Beauregard et al. (2010) se encontró que los delincuentes sexuales que eran jóvenes, especialistas e introvertidos y que seleccionaron a un varón desconocido no procedente de un entorno criminogénico como víctima, eran más propensos a confesar durante el interrogatorio.

3) Confesiones falsas

Una confesión falsa es una admisión detallada de un acto criminal que la persona que confiesa no cometió (Kassin \& Gudjonsson, 2004).

En el estudio de Sigurdsson y Gudjonsson (1996) se encuentra que dentro de los reclusos de la cárceles, las razones más frecuentemente citadas para realizar falsas confesiones fueron librarse de la presión policial (51\%), proteger a otra persona (48\%) y evitar el castigo (40\%).

En el mismo estudio (Sigurdsson \& Gudjonsson, 1996) distinguen entre tres tipos de falsas confesiones: voluntarias, coaccionadas-conformes, coaccionadasinteriorizadas:

Voluntarias. En ocasiones personas inocentes, sin demasiada presión de la policía, realizan confesiones voluntarias falsas. Las causas pueden ser un deseo de notoriedad, proteger a otra persona o la incapacidad de distinguir la realidad de la fantasía.

Coaccionadas-conformes. En ellas los sospechosos son inducidos a confesar un crimen que no cometieron a lo largo del interrogatorio. En este caso, el sujeto se presta a la confesión para escapar de una situación adversa, evitar una amenaza explícita o implícita o para ganar una recompensa explícita o implícita.

Coaccionadas-interiorizadas. En estas, personas inocentes y vulnerables bajo la influencia de técnicas de interrogatorio altamente sugestivas, no solamente 
cambian su comportamiento sino que creen que cometieron el crimen en cuestión (a veces confabulando memorias falsas en el proceso).

En otro estudio (Gudjonsson et al., 2008) se muestra la importancia del factor "proteger a un amigo" dentro de las razones para realizar una falsa confesión entre jóvenes delincuentes (como una muestra de lealtad grupal).

En el estudio de Gudjonsson et al. (2006) las confesiones falsas durante el interrogatorio fueron asociadas principalmente con el nivel de implicación de actividades delictivas, implicación de amigos y de presión.

Personalidad en las confesiones falsas. Según estudio de Sigurdsson y Gudjonsson (1996) realizado entre reclusos, las confesiones falsas en Islandia se circunscriben a delincuentes reincidentes con rasgos de personalidad antisocial. Esta circunstancia puede deberse a la mayor impulsividad de este tipo de personalidades que puede aparecer en el interrogatorio ante la presión de la policía y a que tienen una menor preocupación o miedo de las consecuencias.

En otro estudio (Gudjonsson et al., 2008) se observó que tener un historial siendo víctima de abusos influye en la vulnerabilidad del sujeto.

Las personas con mayor respeto a la autoridad se muestran más sensibles a las preguntas directivas por lo que son más propensas a cambiar sus respuestas como reacción al feedback negativo. Así mismo, las personas más sensibles a la preguntas directivas fueron más propensas a creer que el azar es el que controla sus vidas (Forrest, Wadkins, \& Larson, 2006).

Según Kassin y Gudjonsson (2004) las personas responden de manera diferente ante la presión del interrogatorio. En concreto, aquellas personas más sumisas o que tratan de agradar en situaciones sociales serán más vulnerables en el proceso de interrogatorio.

\section{Factores de riesgo de las confesiones falsas.}

Además de algunos factores que hemos visto previamente y que recordaremos, existen factores que incrementan la vulnerabilidad del sujeto y por tanto el riesgo de realizar una confesión falsa:

- Juventud: cuanto más joven el sujeto más riesgo de verse influenciado por ejemplo, por una figura de autoridad, preguntas directivas o presión social (Kassin \& Gudjonsson, 2004).

- Retraso mental o discapacidad intelectual y del desarrollo. Estos sujetos son particularmente vulnerables porque además son influenciados más fácilmente por figuras de autoridad (Kassin \& Gudjonsson, 2004).

- Personas con psicopatologías, memorias o percepciones distorsionadas. Pueden llevar a los sujetos a información equivocada que resulte en confesiones falsas (Kassin \& Gudjonsson, 2004).

- Aislamiento y custodia física (Kassin \& Gudjonsson, 2004; Russano et al., 2005).

- Confrontación en el interrogatorio (Kassin \& Gudjonsson, 2004; Le Bihan \& Bénézech, 2013; Russano et al., 2005).

- Técnicas de maximización y minimización (Russano et al., 2005).

- Preferencia de las consecuencias a corto plazo frente al largo plazo (Madon et al., 2012).

- Efectos del síndrome de desconfianza de la memoria (Gudjonsson, 2016).

- Efectos del feedback negativo (Baxter et al., 2006; McMurtrie et al., 2012; Tata \& Gudjonsson, 1990).

4) Confesiones en los tribunales

Estudios (Kassin \& Gudjonsson, 2004; Kassin \& Neumann, 1997) han revelado la importancia que tienen las confesiones en los tribunales por encima de otras pruebas como por ejemplo, los testimonios de testigos presenciales. Así mismo se ha investigado la importancia del impacto de la actitud no verbal de los testigos en las deliberaciones de los jurados (Newcombe \& Bransgrove, 2007).

Sin embargo, los jurados tienden a creer en la veracidad de las falsas confesiones al igual que lo han hecho antes en el proceso los investigadores. En un estudio realizado (Leo \& Liu, 2009) con jurados simulados se observa que a pesar de que estos creen en la capacidad de las técnicas coercitivas de interrogatorio para obtener confesiones verdaderas en ocasiones, no 
reconocen que esas mismas técnicas puedan desembocar en confesiones falsas, pensamiento contrario a la evidencia científica disponible. En ese mismo estudio se presentan los argumentos científicos para que testigos expertos puedan ayudar a los jurados a entender las implicaciones de determinadas técnicas de interrogatorio y cómo estas pueden resultar en falsas confesiones.

\section{CONCLUSIONES}

Como se ha observado en la revisión, gran cantidad de las prácticas "persuasivas" u orientadas a conseguir la confesión del sospechoso, tienen el riesgo de conseguirla no sólo de sujetos culpables sino también de inocentes. El aislamiento del entorno familiar o la incomodidad en la entrevista, la confrontación con la policía o con evidencias, las técnicas de minimización del delito que pueden sugerir una indulgencia implícita, etc. son factores que pueden llevar a la persona interrogada a tratar de escapar de la situación realizando la confesión que se le pide. En algunos países como Reino Unido se han dado pasos desde hace tiempo para incrementar la protección de los sospechosos y minimizar los riesgos de condenar a un inocente por una confesión falsa resultante de un proceso sin garantías (ver Police and Criminal Evidence Act (PACE), 1984). Es necesario también consolidar la protección de los derechos de los sospechosos una vez ante los tribunales (con una mayor difusión de los estudios científicos que explican los peligros de confesiones falsas, con la labor didáctica de testigos expertos ante casos con jurados, etc.).

El acceso a la técnica y a las nuevas tecnologías puede servir de ayuda para garantizar el proceso. Un ejemplo es la grabación en video de los interrogatorios y la digitalización de los mismos, así como del material relacionado con los casos. En la medida que ello sea posible en todos los procesos, por un lado, se evitarán posibles abusos o malas prácticas que pudieran darse de no existir los registros, a la vez que facilitaría la labor del interrogador pudiendo este centrarse en el proceso mientras está con el sujeto y completar con posterioridad el análisis tras el visionado de la entrevista y de todo el material relevante.
Es necesario seguir profundizando en el estudio de técnicas persuasivas que sean capaces de obtener información fiable disminuyendo al máximo posible los riesgos de técnicas coercitivas (por ejemplo técnicas de "desafío suave", entrevista cognitiva, técnica SUE (Strategic Use of Evidence) (Hartwig, Granhag, Stromwall, \& Kronkvist, 2006), etc.). Sería igualmente positivo el desarrollo y estudio de impacto y efectividad de adaptaciones de otros ámbitos como por ejemplo, el Aikido (Chew, 1995).

La evolución de la persuasión en el interrogatorio no puede ser vista como un solo elemento sobre el que intervenir sino como un sistema interrelacionado donde hay que actuar en varios ámbitos de una manera coordinada (Gudjonsson, 2003; Kassin \& Gudjonsson, 2004):

- La custodia policial (aislamiento, técnicas interrogatorio, etc.).

- La personalidad y comportamiento del investigador.

- Las características específicas del sujeto (personalidad, edad, inteligencia, predisposición a la sugestión) y del crimen (notoriedad, respuestas del acusado).

- Impacto de consejero legal.

No se ha profundizado en esta revisión en los aspectos referentes a la personalidad de los entrevistadores ni de los entrevistados pero en este campo se pueden conseguir avances significativos de conocimiento que permitan mejorar los procesos de entrevista.

Otro elemento crítico para que una vez definido el proceso óptimo, sea llevado a cabo en las mejores condiciones, es el entrenamiento de las personas destinadas a realizar la entrevista. Dado el número de variables que pueden intervenir, sólo mediante entrenamiento exhaustivo y la acumulación de experiencia podrán obtenerse progresivamente mejores resultados, lo que sugiere que esta labor de entrevistador se especialice más en el futuro. 


\section{REFERENCIAS}

Alison, L., Alison, E., Noone, G., Elntib, S., Waring, S., \& Christiansen, P. (2014). Whatever you say, say nothing: Individual differences in counter interrogation tactics amongst a field sample of right wing, AQ inspired and paramilitary terrorists. Personality and Individual Differences, 68, 170-175. http://doi.org/10.1016/j.paid.2014.04.031

Baxter, J. S., Boon, J. C. W., \& Marley, C. (2006). Interrogative pressure and responses to minimally leading questions. Personality and Individual Differences, 40(1), 87-98. http://doi.org/10.1016/j.paid.2005.06.017

Beauregard, E., Deslauriers-Varin, N., \& St-Yves, M. (2010). Interactions between factors related to the decision of sex offenders to confess during police interrogation: a classification-tree approach. Sexual Abuse: A Journal of Research and Treatment, 22(3), 343-367. Journal Article, Research Support, Non-U.S. Gov't. http://doi.org/10.1177/1079063210370707

Chew, P. G. L. (1995). Aikido politics in interview interaction. Linguistics and Education, 7(3), 201-220. JOUR. http://doi.org/http://dx.doi.org/10.1016/08985898(95)90023-3

Choe, D. H. (2013). The prosecutor's interview with suspects in South Korea. International Journal of Law, Crime and Justice, 41(4), 322-342. http://doi.org/10.1016/j.ijlcj.2013.07.007

Cialdini, R. B. (1984). Influence. The psychology of persuasion. New York.

Davison, S. E., \& Gossop, M. (1999). The Management of Opiate Addicts in Police Custody. Medicine, Science and the Law , 39(2), 153-160.

JOUR. http://doi.org/10.1177/002580249903900210

Diccionario de la lengua española (RAE). (2016). Retrieved from http://dle.rae.es/?id=SkN2E1W

Fisher, R. P., \& Geiselman, R. E. (2010). The Cognitive Interview method of conducting police interviews: Eliciting extensive information and promoting Therapeutic Jurisprudence. International Journal of Law and Psychiatry, 33(5), 321-328. http://doi.org/10.1016/j.ijlp.2010.09.004

Fisher, R. P., Geiselman, R. E., \& Amador, M. (1989). Field test of the Cognitive Interview: enhancing the recollection of actual victims and witnesses of crime. The Journal of Applied Psychology, 74(5), 722-727. Journal Article, Research Support, U.S. Gov't, Non-P.H.S.

Forrest, K. D., Wadkins, T. A., \& Larson, B. A. (2006). Suspect personality, police interrogations, and false confessions: Maybe it is not just the situation. Personality and Individual Differences, 40(3), 621-628. JOUR. http://doi.org/http://dx.doi.org/10.1016/j.paid.2005.09.002

Gaines, P. (2011). The Multifunctionality of Discourse Operator Okay: Evidence from a police interview. Journal of Pragmatics, 43(14), 3291-3315. JOUR. http://doi.org/http://dx.doi.org/10.1016/j.pragma.2011.06.0 05
Gall, J. A. M., \& Freckelton, I. (1999). Fitness for interview: current trends, views and an approach to the assessment procedure. Journal of Clinical Forensic Medicine, 6(4), 213-223. JOUR. http://doi.org/http://dx.doi.org/10.1016/S13531131(99)90000-7

González, J. L. (2009). La entrevista policial: aspectos generales. In Manual de Investigación Policial, módulo 6: Técnicas indagatorias (pp. 569-580). (Gobierno de Canarias): Thomson Reuters.

Gudjonsson, G. (2016). Memory distrust syndrome, confabulation and false confession. Cortex. JOUR. http://doi.org/http://dx.doi.org/10.1016/j.cortex.2016.06.01 3

Gudjonsson, G. H. (2003). Psychology brings justice: the science of forensic psychology. Criminal Behaviour and Mental Health: CBMH, 13(3), 159-167. Journal Article.

Gudjonsson, G. H. (2006). Sex offenders and confessions: How to overcome their resistance during questioning. Journal of Clinical Forensic Medicine, 13(4), 203-207. JOUR. http://doi.org/http://dx.doi.org/10.1016/j.jcfm.2006.02.003

Gudjonsson, G. H., \& Petursson, H. (1991). Custodial interrogation: Why do suspects confess and how does it relate to their crime, attitude and personality? Personality and Individual Differences, 12(3), 295-306. http://doi.org/10.1016/01918869(91)90116-S

Gudjonsson, G. H., \& Sigurdsson, J. F. (1999). The Gudjonsson Confession Questionnaire-Revised (GCQ-R) factor structure and its relationship with personality. Personality and Individual Differences, $\quad 27(5), \quad$ 953-968. JOUR. http://doi.org/http://dx.doi.org/10.1016/S0191$8869(98) 00278-5$

Gudjonsson, G. H., Sigurdsson, J. F., Asgeirsdottir, B. B., \& Sigfusdottir, I. D. (2006). Custodial interrogation, false confession and individual differences: A national study among Icelandic youth. Personality and Individual Differences, 41(1), 4959. http://doi.org/10.1016/j.paid.2005.12.012

Gudjonsson, G. H., Sigurdsson, J. F., Sigfusdottir, I. D., \& Asgeirsdottir, B. B. (2008). False confessions and individual differences: The importance of victimization among youth. Personality and Individual Differences, 45(8), 801-805. JOUR. http://doi.org/http://dx.doi.org/10.1016/j.paid.2008.08.010

Hartwig, M., Granhag, P. A., Stromwall, L. A., \& Kronkvist, O. (2006). Strategic use of evidence during police interviews: when training to detect deception works. Law and Human Behavior, 30(5), 603-619. Journal Article, Research Support, Non-U.S. Gov't. http://doi.org/10.1007/s10979-006-9053-9

Heydon, G. (2011). Silence: Civil right or social privilege? A discourse analytic response to a legal problem. Journal of Pragmatics, $\quad 43(9), \quad$ 2308-2316. http://doi.org/10.1016/j.pragma.2011.01.003

Inbau, F. E., Reid, J. E., Buckey, J. P., \& Jayne, B. C. (2001). Criminal interrrogation and confesions (4th ed.). Gaithersburg, MD: Aspen: Jones \& Bartlett Learning. 
Kassin, S. M., Drizin, S. A., Grisso, T., Gudjonsson, G. H., Leo, R. A., \& Redlich, A. D. (2010). Police-induced confessions, risk factors, and recommendations: looking ahead. Law and Human Behavior, 34(1), 49-52. Journal Article. http://doi.org/10.1007/s10979-010-9217-5

Kassin, S. M., \& Gudjonsson, G. H. (2004). The Psychology of Confessions: A Review of the Literature and Issues. Psychological Science in the Public Interest: A Journal of the American Psychological Society, 5(2), 33-67. Journal Article. http://doi.org/10.1111/j.1529-1006.2004.00016.x

Kassin, S. M., \& Neumann, K. (1997). On the Power of Confession Evidence: An Experimental Test of the Fundamental Difference Hypothesis. Law and Human Behavior, 21(5), 469484. article. http://doi.org/10.1023/A:1024871622490

Launay, C., \& Py, J. (2015). Methods and aims of investigative interviewing of adult witnesses: An analysis of professional practices. Pratiques Psychologiques, 21(1), 55-70. JOUR. http://doi.org/http://dx.doi.org/10.1016/j.prps.2014.11.001

Le Bihan, P., \& Bénézech, M. (2013). Psychologie des faux aveux : données classiques et contemporaines. Annales MédicoPsychologiques, Revue Psychiatrique, 171(7), 468-475. JOUR. http://doi.org/http://dx.doi.org/10.1016/j.amp.2013.05.009

Leo, R. A., \& Liu, B. (2009). What do potential jurors know about police interrogation techniques and false confessions? Behavioral Sciences \& the Law, 27(3), 381-399. Journal Article. http://doi.org/10.1002/bsl.872

Madon, S., Guyll, M., Scherr, K. C., Greathouse, S., \& Wells, G. L. (2012). Temporal discounting: The differential effect of proximal and distal consequences on confession decisions. Law and Human Behavior. JOUR PMID - 22471381, Madon, Stephanie: Department of Psychology, Iowa State University, W112 Lagomarcino Hall, Ames, IA, US, 50011, madon@iastate.edu: Educational Publishing Foundation. http://doi.org/10.1037/h0093962

Mason, M. (2016). The "preparatory" and "argumentation" stages of police interrogation: A linguistic analysis of a criminal investigation. Language \& Communication, 48, 79-87. JOUR. http://doi.org/http://dx.doi.org/10.1016/j.langcom.2016.03. 001

McMurtrie, H., Baxter, J. S., Obonsawin, M. C., \& Hunter, S. C. (2012). Consistent witness responses: The effects of age and negative feedback. Personality and Individual Differences, 53(8), 958-962. http://doi.org/10.1016/j.paid.2012.07.008

Nakane, I. (2011). The role of silence in interpreted police interviews. Journal of Pragmatics, 43(9), 2317-2330. JOUR. http://doi.org/http://dx.doi.org/10.1016/j.pragma.2010.11.0 13

Newcombe, P. A., \& Bransgrove, J. (2007). Perceptions of witness credibility: Variations across age. Journal of Applied Developmental Psychology, 28(4), 318-331. JOUR. http://doi.org/http://dx.doi.org/10.1016/j.appdev.2007.04.0 03

Police and Criminal Evidence Act (PACE). (1984).
PubMed. (2016). $\quad$ Retrieved from https://es.wikipedia.org/wiki/PubMed

Rix, K. J. . (1998). Silence in interview: psychiatry and the Argent conditions. Journal of Clinical Forensic Medicine (Vol. 5). http://doi.org/10.1016/S1353-1131(98)90135-3

Russano, M. B., Meissner, C. A., Narchet, F. M., \& Kassin, S. M. (2005). Investigating true and false confessions within a novel experimental paradigm. Psychological Science, 16(6), 481-486. Journal Article. http://doi.org/10.1111/j.09567976.2005.01560.x

Science Direct. (2016). Retrieved from http://www.americalatina.elsevier.com/corporate/es/science _direct.php

Sigurdsson, J. F., \& Gudjonsson, G. H. (1994). Alcohol and drug intoxication during police interrogation and the reasons why suspects confess to the police. Addiction, 89(8), 985-997. http://doi.org/10.1111/j.1360-0443.1994.tb03358.x

Sigurdsson, J. F., \& Gudjonsson, G. H. (1996). The psychological characteristics of "false confessors". A study among icelandic prison inmates and juvenile offenders. Personality and Individual Differences, 20(3), 321-329. http://doi.org/10.1016/01918869(95)00184-0

Tata, P. R., \& Gudjonsson, G. H. (1990). The effects of mood and verbal feedback on interrogative suggestibility. Personality and Individual Differences, 11(10), 1079-1085. JOUR. http://doi.org/http://dx.doi.org/10.1016/01918869(90)90136-F

Vernham, Z., Vrij, A., Leal, S., Mann, S., \& Hillman, J. (2014). Collective interviewing: A transactive memory approach towards identifying signs of truthfulness. Journal of Applied Research in Memory and Cognition, 3(1), 12-20. JOUR. http://doi.org/http://dx.doi.org/10.1016/j.jarmac.2014.01.0 01

Vrij, A., Mann, S., \& Fisher, R. P. (2006). Information-gathering vs accusatory interview style: Individual differences in respondents' experiences. Personality and Individual Differences, 41(4), 589-599. http://doi.org/10.1016/j.paid.2006.02.014

Walton, D. (2003). The interrogation as a type of dialogue. Journal of Pragmatics, 35(12), 1771-1802. http://doi.org/10.1016/S03782166(02)00201-1

Wu, W., \& Vander Beken, T. (2012). The evolution of criminal interrogation rules in China. International Journal of Law, Crime and Justice, 40(4), 271-295. http://doi.org/10.1016/j.ijlcj.2011.09.007

Yuille, J. C., Marxsen, D., \& Cooper, B. (1999). Training Investigative Interviewers: Adherence to the Spirit, As Well As the Letter. International Journal of Law and Psychiatry, 22(3), 323-336. http://doi.org/10.1016/S0160-2527(99)00012-6. 


\section{ANEXO}

Tabla 3. Relación de artículos de la revisión sistemática

\begin{tabular}{|c|c|c|c|}
\hline Búsqueda & número & Autor y Título & Año \\
\hline B1 SD & 1 & $\begin{array}{l}\text { Alison, L., Alison, E., Noone, G., Elntib, S., Waring, S., \& Christiansen, P. (2014). Whatever you } \\
\text { say, say nothing: Individual differences in counter interrogation tactics amongst a field sample of } \\
\text { right wing, AQ inspired and paramilitary terrorists. Personality and Individual Differences, } 68 \text {, } \\
170-175 .\end{array}$ & 2014 \\
\hline B1 SD & 2 & $\begin{array}{l}\text { Baxter, J. S., Boon, J. C. W., \& Marley, C. (2006). Interrogative pressure and responses to minimally } \\
\text { leading questions }\end{array}$ & 2006 \\
\hline B1 SD & 3 & Chew, P. G. L. (1995). Aikido politics in interview interaction. & 1995 \\
\hline B1 SD & 4 & Choe, D. H. (2013). The prosecutor's interview with suspects in South Korea. & 2013 \\
\hline B1 SD & 5 & $\begin{array}{l}\text { Fisher, R. P., \& Geiselman, R. E. (2010). The Cognitive Interview method of conducting police } \\
\text { interviews: Eliciting extensive information and promoting Therapeutic Jurisprudence }\end{array}$ & 2010 \\
\hline B1 SD & 6 & $\begin{array}{l}\text { Gall, J. A. M., \& Freckelton, I. (1999). Fitness for interview: current trends, views and an approach } \\
\text { to the as sessment procedure }\end{array}$ & 1999 \\
\hline B1 SD & 7 & $\begin{array}{l}\text { Gudjonsson, G. H., \& Petursson, H. (1991). Custodial interrogation: Why do suspects confess and } \\
\text { how does it relate to their crime, attitude and personality? }\end{array}$ & 1991 \\
\hline B1 SD & 8 & $\begin{array}{l}\text { Gudjonsson, G. H., Sigurdsson, J. F., Asgeirsdottir, B. B., \& Sigfusdottir, I. D. (2006). Custodial } \\
\text { interrogation, false confession and individual differences: A national study among Icelandic youth }\end{array}$ & 2006 \\
\hline B1 SD & & $\begin{array}{l}\text { Gudjonsson, G. H., \& Sigurdsson, J. F. (1999). The Gudjonsson Confession Questionnaire-Revised } \\
\text { (GCQ-R) factor structure and its relationship with personality. Personality and Individual } \\
\text { Differences, 27(5), 953-968. }\end{array}$ & 1999 \\
\hline B1 SD & 10 & $\begin{array}{l}\text { Heydon, G. (2011). Silence: Civil right or social privilege? A discourse analytic response to a legal } \\
\text { problem. }\end{array}$ & 2011 \\
\hline B1 SD & 11 & $\begin{array}{l}\text { Launay, C., \& Py, J. (2015). Methods and aims of investigative interviewing of adult witnesses: An } \\
\text { analys is of professional practices. Pratiques Psychologiques, 21(1), }\end{array}$ & 2015 \\
\hline B1 SD & 12 & $\begin{array}{l}\text { Mason, M. (2016). The "preparatory" and "argumentation" stages of police interrogation: A } \\
\text { linguistic analysis of a criminal investigation. }\end{array}$ & 2016 \\
\hline B1 SD & 13 & $\begin{array}{l}\text { McMurtrie, H., Baxter, J. S., Obonsawin, M. C., \& Hunter, S. C. (2012). Consistent witness } \\
\text { responses: The effects of age and negative feedback. }\end{array}$ & 2012 \\
\hline B1 SD & 14 & Nakane, I. (2011). The role of silence in interpreted police interviews & 2011 \\
\hline B1 SD & 15 & Rix, K. J. . (1998). Silence in interview: psychiatry and the Argent conditions & 1998 \\
\hline B1 SD & 16 & $\begin{array}{l}\text { Sigurdsson, J. F., \& Gudjonsson, G. H. (1996). The psychological characteristics of "false } \\
\text { confessors". A study among icelandic prison inmates and juvenile offenders }\end{array}$ & 1996 \\
\hline B1 SD & 17 & $\begin{array}{l}\text { Vernham, Z., Vrij, A., Leal, S., Mann, S., \& Hillman, J. (2014). Collective interviewing: A transactive } \\
\text { memory approach towards identifying signs of truthfulness }\end{array}$ & 2014 \\
\hline B1 SD & 18 & $\begin{array}{l}\text { Vrij, A., Mann, S., \& Fisher, R. P. (2006). Information-gathering vs accusatory interview style: } \\
\text { Individual differences in respondents' experiences }\end{array}$ & 2006 \\
\hline B1 SD & 19 & Wu, W., \& Vander Beken, T. (2012). The evolution of criminal interrogation rules in China & 2012 \\
\hline B1 SD & 20 & $\begin{array}{l}\text { Yuille, J. C., Marxsen, D., \& Cooper, B. (1999). Training Investigative Interviewers: Adherence to } \\
\text { the Spirit, As Well As the Letter }\end{array}$ & 1999 \\
\hline B2 SD & 21 & Gudjonsson, G. (2016). Memory distrust syndrome, confabulation and false confession. & 2016 \\
\hline B3 SD & 22 & $\begin{array}{l}\text { Forrest, K. D., Wadkins, T. A., \& Larson, B. A. (2006). Suspect personality, police interrogations, } \\
\text { and false confessions: Maybe it is not just the situation }\end{array}$ & 2006 \\
\hline B3 SD & 23 & $\begin{array}{l}\text { Gudjonsson, G. H. (2006). Sex offenders and confessions: How to overcome their res is tance during } \\
\text { questioning }\end{array}$ & 2006 \\
\hline B3 SD & 24 & $\begin{array}{l}\text { Gudjonsson, G. H., Sigurdsson, J. F., Sigfusdottir, I. D., \& Asgeirsdottir, B. B. (2008). .False } \\
\text { confessions and individual differences: The importance of victimization among youth }\end{array}$ & 2008 \\
\hline B3 SD & 25 & $\begin{array}{l}\text { Le Bihan, P., \& Bénézech, M. (2013). Psychologie des faux aveux: données classiques et } \\
\text { contemporaines }\end{array}$ & 2013 \\
\hline B3 SD & 26 & $\begin{array}{l}\text { Tata, P. R., \& Gudjonsson, G. H. (1990). The effects of mood and verbal feedback on interrogative } \\
\text { suggestibility. Personality and Individual Differences, } 11(10), 1079-\end{array}$ & 1990 \\
\hline B4 SD & 27 & Newcombe, P. A., \& Brans grove, J. (2007). Perceptions of witness credibility: Variations across age & 2007 \\
\hline B4 SD & 28 & $\begin{array}{l}\text { Gaines, P. (2011). The Multifunctionality of Discourse Operator Okay: Evidence from a police } \\
\text { interview }\end{array}$ & 2008 \\
\hline B1 PM & 29 & Davis on SE, Gossop M.(1999).The management of opiate addicts in police custody. & 1999 \\
\hline B1 PM & 30 & $\begin{array}{l}\text { Sigurdsson JF, Gudjonsson GH.Addiction. 1994. Alcohol and drug intoxication during police } \\
\text { interrogation and the reasons why suspects confess to the police. }\end{array}$ & 1994 \\
\hline B2 PM & 31 & $\begin{array}{l}\text { Madon S, Guyll M, Scherr KC, Greathouse S, Wells GL (2012). Temporal discounting: the } \\
\text { differential effect of proximal and distal consequences on confession decisions }\end{array}$ & 2012 \\
\hline B2 PM & 32 & $\begin{array}{l}\text { Beauregard E, Deslauriers-Varin N, St-Yves M (2010). Interactions between factors related to the } \\
\text { decision of sex offenders to confess during police interrogation: a classification-tree approach. }\end{array}$ & 2010 \\
\hline B2 PM & 33 & $\begin{array}{l}\text { Russano MB, Meissner CA, Narchet FM, Kassin SM. (2005).Investigating true and false } \\
\text { confessions within a novel experimental paradigm. }\end{array}$ & 2005 \\
\hline B2 PM & 34 & $\begin{array}{l}\text { Kassin SM, Gudjonsson GH (2004). The Psychology of Confessions: A Review of the Literature } \\
\text { and Issues. }\end{array}$ & 2004 \\
\hline B4 PM & 35 & $\begin{array}{l}\text { Leo RA, Liu B. (2009)What do potential jurors know about police interrogation techniques and } \\
\text { false confessions? }\end{array}$ & 2009 \\
\hline
\end{tabular}

